PROCEEDINGS OF THE

AMERICAN MATHEMATICAL SOCIETY

Volume 132, Number 12, Pages 3485-3490

S 0002-9939(04)07658-0

Article electronically published on July 22, 2004

\title{
LOCAL COHOMOLOGY MODULES WITH INFINITE DIMENSIONAL SOCLES
}

\author{
THOMAS MARLEY AND JANET C. VASSILEV
}

(Communicated by Bernd Ulrich)

\begin{abstract}
In this paper we prove the following generalization of a result of Hartshorne: Let $T$ be a commutative Noetherian local ring of dimension at least two, $R=T\left[x_{1}, \ldots, x_{n}\right]$, and $I=\left(x_{1}, \ldots, x_{n}\right)$. Let $f$ be a homogeneous element of $R$ such that the coefficients of $f$ form a system of parameters for $T$. Then the socle of $H_{I}^{n}(R / f R)$ is infinite dimensional.
\end{abstract}

\section{INTRODUCTION}

The third of Huneke's four problems in local cohomology $[\mathrm{Hu}]$ is to determine when $H_{I}^{i}(M)$ is Artinian for a given ideal $I$ of a commutative Noetherian local ring $R$ and finitely generated $R$-module $M$. An $R$-module $N$ is Artinian if and only if $\operatorname{Supp}_{R} N \subseteq\{m\}$ and $\operatorname{Hom}_{R}(R / m, N)$ is finitely generated, where $m$ is the maximal ideal of $R$. Thus, Huneke's problem may be separated into two subproblems:

- When is $\operatorname{Supp}_{R} H_{I}^{i}(M) \subseteq\{m\}$ ?

- When is $\operatorname{Hom}_{R}\left(R / m, H_{I}^{i}(M)\right)$ finitely generated?

This article is concerned with the second question. For an $R$-module $N$, one may identify $\operatorname{Hom}_{R}(R / m, N)$ with the submodule $\{x \in N \mid m x=0\}$, which is an $R / m$-vector space called the socle of $N$ (denoted $\operatorname{soc}_{R} N$ ). It is known that if $R$ is an unramified regular local ring, then the local cohomology modules $H_{I}^{i}(R)$ have finite dimensional socles for all $i \geq 0$ and all ideals $I$ of $R$ ([HS], [L1], [L2]). The first example of a local cohomology module with an infinite dimensional socle was given in 1970 by Hartshorne [Ha]: Let $k$ be a field, $R=k[[u, v]][x, y]$, $P=(u, v, x, y) R, I=(x, y) R$, and $f=u x+v y$. Then $\operatorname{soc}_{R_{P}} H_{I R_{P}}^{2}\left(R_{P} / f R_{P}\right)$ is infinite dimensional. Of course, since $I$ and $f$ are homogeneous, this is equivalent to saying that $\operatorname{Hom}_{R}\left(R / P, H_{I}^{2}(R / f R)\right)$ (the * socle of $\left.H_{I}^{2}(R / f R)\right)$ is infinite dimensional. Hartshorne proved this by exhibiting an infinite set of linearly independent elements in the * socle of $H_{I}^{2}(R)$.

In the last 30 years there have been few results in the literature which explain or generalize Harthshorne's example. For affine semigroup rings, a remarkable result proved by Helm and Miller [HM] gives necessary and sufficient conditions (on the semigroup) for the ring to possess a local cohomology module (of a finitely generated

Received by the editors July 16, 2003.

2000 Mathematics Subject Classification. Primary 13D45.

Key words and phrases. Local cohomology.

The first author was partially supported by NSF grant DMS-0071008.

(C)2004 American Mathematical Society Reverts to public domain 28 years from publication 
module) having infinite dimensional socle. Beyond that work, however, little has been done.

In this paper we prove the following:

Theorem 1.1. Let $(T, m)$ be a Noetherian local ring of dimension at least two. Let $R=T\left[x_{1}, \ldots, x_{n}\right]$ be a polynomial ring in $n$ variables over $T, I=\left(x_{1}, \ldots, x_{n}\right)$, and $f \in R$ a homogeneous polynomial whose coefficients form a system of parameters for $T$. Then the * socle of $H_{I}^{n}(R / f R)$ is infinite dimensional.

Hartshorne's example is obtained by letting $T=k[[u, v]], n=2$, and $f=u x+v y$ (homogeneous of degree 1). Note, however, that we do not require the coefficient ring to be regular, or even Cohen-Macaulay. As a further illustration, consider the following:

Example 1.2. Let $R=k\left[\left[u^{4}, u^{3} v, u v^{3}, v^{4}\right]\right][x, y, z], I=(x, y, z) R$, and $f=u^{4} x^{2}+$ $v^{8} y z$. Then the * socle of $H_{I}^{3}(R / f R)$ is infinite dimensional.

Part of the proof of Theorem 1.1 was inspired by the recent work of Katzman Ka where information on the graded pieces of $H_{I}^{n}(R / f R)$ is obtained by examining matrices of a particular form. We apply this technique in the proof of Lemma 2.8

Throughout, all rings are assumed to be commutative with identity. The reader should consult [Mat] or [BH] for any unexplained terms or notation and [BS] for the basic properties of local cohomology.

\section{THE MAIN RESULT}

Let $R=\bigoplus R_{\ell}$ be a Noetherian ring graded by the nonnegative integers. Assume $R_{0}$ is local and let $P$ be the homogeneous maximal ideal of $R$. Given a finitely generated graded $R$-module $M$ we define the ${ }^{*}$ socle of $M$ by

$$
\begin{aligned}
{ }^{*} \operatorname{soc}_{R} M & =\{x \in M \mid P x=0\} \\
& \cong \operatorname{Hom}_{R}(R / P, M) .
\end{aligned}
$$

Clearly, ${ }^{*} \operatorname{soc}_{R} M \cong \operatorname{soc}_{R_{P}} M_{P}$. An interesting special case of Huneke's third problem is the following:

Question 2.1. Let $n:=\mu_{R}\left(R_{+} / P R_{+}\right)$, the minimal number of generators of $R_{+}$. When is ${ }^{*} \operatorname{soc} H_{R_{+}}^{n}(R)$ finitely generated?

For $i \in \mathbb{N}$ it is well known that $H_{R+}^{i}(R)$ is a graded $R$-module, each graded piece $H_{R_{+}}^{i}(R)_{\ell}$ is a finitely generated $R_{0}$-module, and $H_{R_{+}}^{i}(R)_{\ell}=0$ for all sufficiently large integers $\ell([\mathrm{BS}, 15.1 .5])$. If we know a priori that $H_{R+}^{n}(R)_{\ell}$ has finite length for all $\ell$ (e.g., if $\operatorname{Supp}_{R} H_{R_{+}}^{n}(R) \subseteq\{P\}$ ), then Question 2.1 is equivalent to:

Question 2.2. When is $\operatorname{Hom}_{R}\left(R / R_{+}, H_{R_{+}}^{n}(R)\right)$ finitely generated?

We give a partial answer to these questions for hypersurfaces. For the remainder of this section we adopt the following notation: Let $(T, m)$ be a local ring of dimension $d$ and $R=T\left[x_{1}, \ldots, x_{n}\right]$ a polynomial ring in $n$ variables over $T$. We endow $R$ with an $\mathbb{N}$-grading by setting $\operatorname{deg} T=0$ and $\operatorname{deg} x_{i}=1$ for all $i$. Let $I=R_{+}=\left(x_{1}, \ldots, x_{n}\right) R$ and let $P=m+I$ be the homogeneous maximal ideal of $R$. Let $f \in R$ be a homogeneous element of degree $p$ and $C_{f}$ the ideal of $T$ generated by the coefficients of $f$. 
Our main result is the following:

Theorem 2.3. Assume $d \geq 2$ and the (nonzero) coefficients of $f$ form a system of parameters for $T$. Then ${ }^{*} \operatorname{soc}_{R} H_{I}^{n}(R / f R)$ is not finitely generated.

The proof of this theorem will be given in a series of lemmas below. Before proceeding with the proof we make a couple of remarks:

Remark 2.4. (a) If $d \leq 1$ in Theorem 2.3, then ${ }^{*} \operatorname{soc} H_{I}^{n}(R / f R)$ is finitely generated. This follows from [DM, Corollary 2] since $\operatorname{dim} R / I=\operatorname{dim} T \leq 1$.

(b) The hypothesis that the nonzero coefficients of $f$ form a system of parameters for $T$ is stronger than our proof requires. One only needs that $C_{f}$ be $m$ primary and that there exists a dimension 2 ideal containing all but two of the coefficients of $f$. (See the proof of Lemma 2.8])

The following lemma identifies the support of $H_{I}^{n}(R / f R)$ for a homogeneous element $f \in R$. This lemma also follows from a much more general result recently proved by Katzman and Sharp [KS, Theorem 1.5].

Lemma 2.5. Let $f \in R$ be a homogeneous element. Then

$$
\operatorname{Supp}_{R} H_{I}^{n}(R / f R)=\left\{Q \in \operatorname{Spec} R \mid Q \supseteq I+C_{f}\right\} .
$$

Proof. It is enough to prove that $H_{I}^{n}(R / f R)=0$ if and only if $C_{f}=T$. As $H_{I}^{n}(R / f R)_{k}$ is a finitely generated $T$-module for all $k$, we have by Nakayama that $H_{I}^{n}(R / f R)=0$ if and only if $H_{I}^{n}(R / f R) \otimes_{T} T / m=0$. Now

$$
\begin{aligned}
H_{I}^{n}(R / f R) \otimes_{T} T / m & \cong H_{I}^{n}\left(R / f R \otimes_{T} T / m\right) \\
& \cong H_{N}^{n}(S / f S)
\end{aligned}
$$

where $S=(T / m)\left[x_{1}, \ldots, x_{n}\right]$ is a polynomial ring in $n$ variables over a field and $N=\left(x_{1}, \ldots, x_{n}\right) S$. As $\operatorname{dim} S=n$, we see that $H_{N}^{n}(S / f S)=0$ if and only if the image of $f$ modulo $m$ is nonzero. Hence, $H_{I}^{n}(R / f R)=0$ if and only if at least one coefficient of $f$ is a unit, i.e., $C_{f}=T$.

We are mainly interested in the case when the coefficients of $f$ generate an $m$-primary ideal:

Corollary 2.6. Let $f \in R$ be homogeneous and suppose $C_{f}$ is m-primary. Then

$$
\operatorname{Supp}_{R} H_{I}^{n}(R / f R)=\{P\} .
$$

Our next lemma is the key technical result in the proof of Theorem 2.3 .

Lemma 2.7. Suppose $u, v \in T$ such that $\operatorname{ht}(u, v) T=2$. For each integer $n \geq 1$ let $M_{n}$ be the cokernel of $\phi_{n}: T^{n+1} \rightarrow T^{n}$ where $\phi_{n}$ is represented by the matrix

$$
A_{n}=\left(\begin{array}{ccccccc}
u & v & 0 & 0 & \cdots & 0 & 0 \\
0 & u & v & 0 & \cdots & 0 & 0 \\
0 & 0 & u & v & \cdots & 0 & 0 \\
\vdots & \vdots & \vdots & \ddots & \ddots & \vdots & \vdots \\
0 & 0 & 0 & 0 & \cdots & u & v
\end{array}\right)_{n \times(n+1)}
$$

Let $J=\bigcap_{n \geq 1} \operatorname{ann}_{T} M_{n}$. Then $\operatorname{dim} T / J=\operatorname{dim} T$. 
Proof. Let $\hat{T}$ denote the $m$-adic completion of $T$. Then $\operatorname{ht}(u, v) \hat{T}=2, \operatorname{ann}_{T} M_{n}=$ $\operatorname{ann}_{\hat{T}}\left(M_{n} \otimes_{T} \hat{T}\right) \cap T$, and $\operatorname{dim} T /(I \cap T) \geq \operatorname{dim} \hat{T} / I$ for all ideals $I$ of $\hat{T}$. Thus, we may assume $T$ is complete. Now let $p$ be a prime ideal of $T \operatorname{such}$ that $\operatorname{dim} T / p=\operatorname{dim} T$. Since $T$ is catenary, $\operatorname{ht}(u, v) T / p=2$. Assume the lemma is true for complete domains. Then $\bigcap_{n \geq 1} \operatorname{ann}_{T / p}\left(M_{n} \otimes_{T} T / p\right)=p / p$. Hence

$$
\begin{aligned}
J & =\bigcap_{n \geq 1} \operatorname{ann}_{T} M_{n} \\
& \subseteq \bigcap_{n \geq 1} \operatorname{ann}_{T}\left(M_{n} \otimes_{T} T / p\right) \\
& =p,
\end{aligned}
$$

which implies that $\operatorname{dim} T / J \geq \operatorname{dim} T / p=\operatorname{dim} T$. Thus, it suffices to prove the lemma for complete domains.

As $T$ is complete, the integral closure $S$ of $T$ is a finite $T$-module (Mat page 263]). Since $\operatorname{ht}(u, v) S=2$ ([Mat, Theorem 15.6]) and $S$ is normal, $\{u, v\}$ is a regular sequence on $S$. It is easily seen that $I_{n}\left(A_{n}\right)$, the ideal of $n \times n$ minors of $A_{n}$, is $(u, v)^{n} T$. By the main result of $\left.\mathrm{BE}\right]$ we obtain $\operatorname{ann}_{S}\left(M_{n} \otimes_{T} S\right)=(u, v)^{n} S$. Hence $\operatorname{ann}_{T} M_{n} \subseteq(u, v)^{n} S \cap T$. As $S$ is a finite $T$-module, there exists an integer $k$ such that $\operatorname{ann}_{T} M_{n} \subseteq(u, v)^{n-k} T$ for all $n \geq k$. Therefore, $\bigcap_{n \geq 1} \operatorname{ann}_{T} M_{n}=(0)$, which completes the proof.

Lemma 2.8. Assume $d \geq 2$ and let $f \in R$ be a homogeneous element of degree $p$ such that the coefficients of $f$ form a system of parameters for $T$. Then $\operatorname{dim} T / \operatorname{ann}_{T} H_{I}^{n}(R / f R) \geq 2$.

Proof. Let $c_{1}, \ldots, c_{d}$ be the nonzero coefficients of $f$. Let $T^{\prime}=T /\left(c_{3}, \ldots, c_{d}\right) T$ and $R^{\prime}=T^{\prime}\left[x_{1}, \ldots, x_{n}\right] \cong R /\left(c_{3}, \ldots, c_{d}\right) R \cong R \otimes_{T} T^{\prime}$. Since

$$
\begin{aligned}
\operatorname{dim} T / \operatorname{ann}_{T} H_{I}^{n}(R / f R) & \geq \operatorname{dim} T / \operatorname{ann}_{T}\left(H_{I}^{n}(R / f R) \otimes_{T} T^{\prime}\right) \\
& =\operatorname{dim} T^{\prime} / \operatorname{ann}_{T^{\prime}} H_{I R^{\prime}}^{n}\left(R^{\prime} / f R^{\prime}\right),
\end{aligned}
$$

we may assume that $\operatorname{dim} T=2$ and $f$ has exactly two nonzero terms.

For any $w \in R$ there is a surjective map $H_{I}^{n}(R / w f R) \rightarrow H_{I}^{n}(R / f R)$. Hence, $\operatorname{ann}_{T} H_{I}^{n}(R / w f R) \subseteq \operatorname{ann}_{T} H_{I}^{n}(R / f R)$. Thus, we may assume that the terms of $f$ have no (non-unit) common factor. Without loss of generality, we may write $R=T\left[x_{1}, \ldots, x_{k}, y_{1}, \ldots y_{r}\right]$ and $f=u x_{1}^{d_{1}} \cdots x_{k}^{d_{k}}+v y_{1}^{e_{1}} \cdots y_{r}^{e_{r}}=u \mathbf{x}^{\mathbf{d}}+v \mathbf{y}^{\mathbf{e}}$, where $\{u, v\}$ is a system of parameters for $T$. As $f$ is homogeneous, $p=\sum_{i} d_{i}=\sum_{i} e_{i}$.

Applying the right exact functor $H_{I}^{n}(\cdot)$ to $R(-p) \stackrel{f}{\rightarrow} R \rightarrow R / f R \rightarrow 0$ we obtain the exact sequence

$$
H_{I}^{n}(R)_{-\ell-p} \stackrel{f}{\rightarrow} H_{I}^{n}(R)_{-\ell} \rightarrow H_{I}^{n}(R / f R)_{-\ell} \rightarrow 0
$$

for each $\ell \in \mathbb{Z}$. For each $\ell, H_{I}^{n}(R)_{-\ell}$ is a free $T$-module with basis

$$
\left\{\mathbf{x}^{-\alpha} \mathbf{y}^{-\beta} \mid \sum_{i, j} \alpha_{i}+\beta_{j}=\ell, \alpha_{i}>0, \beta_{j}>0 \forall i, j\right\}
$$

(e.g., [BS, Example 12.4.1]). Let $q$ be an arbitrary positive integer and let $\ell(q)=$ $q p+k+r$. Define $L_{-\ell(q)}$ to be the free $T$-summand of $H_{I}^{n}(R)_{-\ell(q)}$ spanned by the set

$$
\left\{\mathbf{x}^{-s \mathbf{d}-\mathbf{1}} \mathbf{y}^{-t \mathbf{e}-\mathbf{1}} \mid s+t=q, s, t \geq 0\right\}
$$


Then the cokernel of $\delta_{q}: L_{-\ell(q+1)} \stackrel{f}{\rightarrow} L_{-\ell(q)}$ is a direct summand (as a $T$-module) of $H_{I}^{n}(R / f R)_{-\ell(q)}$. For a given $q$ we order the basis elements for $L_{-\ell(q)}$ as follows:

$$
x^{-s \mathbf{d}-1} \mathbf{y}^{-t \mathbf{e}-\mathbf{1}}>x^{-s^{\prime} \mathbf{d}-\mathbf{1}} \mathbf{y}^{-t^{\prime} \mathbf{e}-\mathbf{1}}
$$

if and only if $s>s^{\prime}$. With respect to these ordered bases, the matrix representing $\delta_{q}$ is

$$
\left(\begin{array}{ccccccc}
u & v & 0 & 0 & \cdots & 0 & 0 \\
0 & u & v & 0 & \cdots & 0 & 0 \\
0 & 0 & u & v & \cdots & 0 & 0 \\
\vdots & \vdots & \vdots & \ddots & \ddots & \vdots & \vdots \\
0 & 0 & 0 & 0 & \cdots & u & v
\end{array}\right)_{(q+1) \times(q+2)}
$$

By Lemma 2.7] if $J=\bigcap_{q \geq 1} \operatorname{ann}_{T} \operatorname{coker} \delta_{q}$, then $\operatorname{dim} T / J=\operatorname{dim} T=2$. As coker $\delta_{q}$ is a direct $T$-summand of $H_{I}^{n}(R / f R)$, we have $\operatorname{ann}_{T} H_{I}^{n}(R / f R) \subseteq J$. This completes the proof.

Lemma 2.9. Under the assumptions of Lemma 2.8, $\operatorname{Hom}_{R}\left(R / I, H_{I}^{n}(R / f R)\right)$ is not finitely generated as an $R$-module. Consequently, $\operatorname{Hom}_{R}\left(R / I, H_{I}^{n}(R / f R)\right)_{k} \neq 0$ for infinitely many $k$.

Proof. Suppose $\operatorname{Hom}_{R}\left(R / I, H_{I}^{n}(R / f R)\right)$ is finitely generated. By Lemma 3.5 of MV] we have that $I+\operatorname{ann}_{R} H_{I}^{n}(R / f R)$ is $P$-primary. (One should note that the hypothesis in [MV, Lemma 3.5] that the ring be complete is not necessary.) This implies that $\operatorname{ann}_{R} H_{I}^{n}(R / f R) \cap T=\operatorname{ann}_{T} H_{I}^{n}(R / f R)$ is $m$-primary, contradicting Lemma 2.8.

We now give the proof of our main result:

Proof of Theorem 2.3. By Corollary 2.6. $\operatorname{Supp}_{R} H_{I}^{n}(R / f R)=\{P\}$. Thus, $\operatorname{Hom}_{R}\left(R / I, H_{I}^{n}(R / f R)\right)_{k}$ has finite length as a $T$-module for all $k$ and is nonzero for infinitely many $k$ by Lemma 2.9 . Consequently,

$$
\operatorname{Hom}_{R}\left(R / P, H_{I}^{n}(R / f R)\right)_{k}=\operatorname{Hom}_{T}\left(T / m, \operatorname{Hom}_{R}\left(R / I, H_{I}^{n}(R / f R)\right)_{k}\right)
$$

is nonzero for infinitely many $k$. Hence

$$
{ }^{*} \operatorname{Soc}_{R}\left(H_{I}^{n}(R / f R)\right)=\operatorname{Hom}_{R}\left(R / P, H_{I}^{n}(R / f R)\right)
$$

is not finitely generated.

\section{REFERENCES}

[BKS] Brodmann, M., Katzman, M. and Sharp, R., Associated primes of graded component of local cohomology modules, Trans. Amer. Math. Soc., 354, 4261-4283 (2002). MR 1926875 (2003h:13020)

[BS] Brodmann, M. and Sharp, R., Local Cohomology: an algebraic introduction with geometric applications, Cambridge Studies in Advanced Mathematics no. 60, Cambridge, Cambridge University Press, 1998. MR 1613627 (99h:13020)

[BH] Bruns, W. and Herzog, J., Cohen-Macaulay Rings, Cambridge Studies in Advanced Mathematics no. 39, Cambridge, Cambridge University Press, 1993. MR 1251956 (95h:13020)

[BE] Buchsbaum, D. and Eisenbud, D., What annihilates a module?, J. Algebra 47, 231-243 (1977). MR 0476736 (57:16293)

[DM] Delfino, D. and Marley, T., Cofinite modules and local cohomology, J. Pure Appl. Algebra 121, 45-52 (1997). MR 1471123 (98g:13015)

[Ha] Hartshorne, R., Affine Duality and Cofiniteness, Invent. Math. 9, 145-164 (1970). MR 0257096 (41:1750) 
[HM] Helm, D. and Miller, E., Bass numbers of semigroup-graded local cohomology, Pacific J. Math. 209, 41-66 (2003). MR 1973933 (2004c:13028)

[Hu] Huneke, C., Problems on local cohomology, Free Resolutions in commutative algebra and algebraic geometry (Sundance, Utah, 1990), Research Notes in Mathematics 2, Boston, MA, Jones and Bartlett Publishers, 1994, 993-108. MR 1165313 (92m:13001)

[HK] Huneke, C. and Koh, J., Cofiniteness and vanishing of local cohomology modules, Math. Proc. Cambridge Philos. Soc. 110, 421-429 (1991). MR 1120477 (92g:13021)

[HS] Huneke, C. and Sharp, R., Bass Numbers of local cohomology modules, Trans. Amer. Math. Soc. 339, 765-779 (1993). MR 1124167 (93m:13008)

[Ka] Katzman, M., An example of an infinite set of associated primes of a local cohomology module, J. Algebra, 252, 161-166 (2002). MR 1922391 (2003h:13021)

[KS] Katzman, M. and Sharp, R., Some properties of top graded local cohomology modules, J. Algebra 259, 599-612 (2003). MR 1955534 (2004a:13011)

[L1] Lyubeznik, G., Finiteness Properties of local cohomology modules (An application of D-modules to commutative algebra), Invent. Math. 113, 41-55 (1993). MR 1223223 (94e:13032)

[L2] Lyubeznik, G., Finiteness properties of local cohomology modules for regular local rings of mixed characteristic: The unramified case, Comm. Algebra 28 no. 12, 5867-5882 (2000). MR 1808608 (2002b:13028)

[MV] Marley, T. and Vassilev, J., Cofiniteness and associated primes of local cohomology modules, J. Algebra 256, 180-193 (2002). MR 1936885 (2003j:13025)

[Mat] Matsumura, H., Commutative Ring Theory, Cambridge Studies in Advanced Mathematics no. 8, Cambridge, Cambridge University Press, 1986. MR 0879273 (88h:13001)

Department of Mathematics, University of Nebraska-Lincoln, Lincoln, Nebraska 68588-0323

E-mail address: tmarley@math.unl.edu

URL: http://www. math.unl.edu/ ${ }^{\text {tmarley }}$

Department of Mathematical Sciences, University of Arkansas, Fayetteville, ArkanSAS 72701

E-mail address: jvassil@uark.edu

URL: http://comp.uark.edu/ jvassil 